\title{
Partnership through Pandemic
}

\author{
Meghan Engbretson'; Anne Rutherford ${ }^{1}$ \\ ${ }^{1}$ Kingston Health Sciences Centre, Kingston, Ontario, Canada
}

Corresponding author:

Meghan Engbretson, Infection Control Practitioner, Kingston Health Sciences Centre, Kingston, ON, Canada

Tel: 613-549-6666 ext.4457 | meghan.engbretson@kingstonhsc.ca

Distributing sound, evidence-based healthcare guidance can be an uphill battle in a world of media misinformation and hearsay, especially during the current SARS-CoV-2 pandemic, which has pushed the field of infection prevention and control (IPAC) into the spotlight like never before. Faced with the quantity and quality of information - true and false - speeding through healthcare workers, patients and the public, infection control practitioners have scrambled to create, adapt and communicate policies, procedures and protocols to help tackle the new virus.

Never has the need for an adaptive and collaborative approach to communication been more pressing. To offset the risk of disjointed memo trails, outdated webpages and unread emails, the Infection Prevention and Control and Strategy Management and Communications (SMC) teams at Kingston Health Sciences Centre (KHSC) worked together to develop and implement a profile-boosting campaign for IPAC that would help ensure infection control content reached the right audiences through the right channels at the right time.

A research and teaching hospital focused on complex, acute and specialty care, KHSC spreads across two hospital sites (inpatient and ambulatory) and is the workplace of more than 5,000 employees, 600-plus physicians, and 1,000-plus learners. Given the scope of disciplines, practice and practitioners, the IPAC/SMC communications campaign concentrated on recalibrating the internal IPAC brand and communication tools to focus attention on the highly professional, expert IPAC team.

Towards that end, a special IPAC wordmark and IPAC Alert memo template were created to brand internal IPAC communications, and a photo gallery of staff and services was launched to familiarize KHSC staff with IPAC faces and roles. On the hospital intranet, the IPAC site was repositioned at the top of the clinical resources listing, a COVID-19 information hub was folded into the IPAC departmental site and a dedicated departmental email was introduced. This last addition helps to clearly flag important IPAC Alerts that land in staff's inboxes, and provides quick access to IPAC's Infection Control Practitioners. The IPAC team has also expanded its profile while sharing knowledge and insight at a series of all-staff COVID-19 virtual open forums, answering real-time questions on everything from virus prevalence to personal protective equipment (PPE) to testing.

In terms of external communications, the campaign turned to social media to target educational needs via YouTube videos (e.g., donning/doffing PPE, conservation/reuse of PPE, masking, etc.), which helped to establish the IPAC team as players on the national infection control stage. Profiles of IPAC team members have been featured on the \#myKHSC Instagram channel and work is underway to build up a regular Twitter presence. Increasingly, it is clear that the use of social media in healthcare is a critical learning tool. At KHSC, the IPAC/SMC team continues to evaluate and expand IPAC's use of social media to help foster a network of collaborative and evidence-based medicine and to help ensure that KHSC is a reliable and trusted voice for timely and accurate information.

Thanks to the IPAC/SMC collaboration to date, the IPAC at KHSC team has become more recognizable, dependable and approachable to the hospital community at large and the flow of IPAC communication has improved across the organization. It is a collaboration that has laid the groundwork for future opportunities, including multidisciplinary teaching sessions, in-services and information sharing across a growing network of colleagues. The need for a robust infection control program has been clearly demonstrated over the course of the COVID-19 pandemic, and will continue to evolve over time. For that reason, the collaboration stands as a worthwhile investment in patient and staff safety. 